\title{
मानवी विकास आणि सरकारी धोरण
}

\section{Human development and government policy}

Dr. Rakshit Madan Bagde,

Assistant Professor,Late. Mansaramji Padole Arts College, Ganeshpur Bhandara ORCID iD - 0000-0002-7507-0244

Web of Science ResearcherID - AAF-2760-2020

SSRN - Author ID: 4770534

Authenticus ID - R-00J-YM2

Vidwan-ID : 221858

rakshitbagde@gmail.com

\section{प्रस्तावना :-}

UNDP ने सर्वप्रथम 1990 मध्ये अर्थशास्त्रज्ञ महबुब हक यांच्या सहकार्याने मानव विकास रिपोर्ट प्रकाशित केला, या HDI निर्देशांकाचे प्रवर्तक म्हणून त्यांना संबोधले जाते. HDI निर्देशांकातील सर्वाधिक महत्वपुर्ण बाब म्हणजे, दिर्घायुष्य, स्वस्थ जीवन, शिक्षण प्राप्तीची संधी आणि उत्कृष्ट जीवनाचा स्तर तसेच यातील अन्य महत्वाच्या बाबी म्हणजे राजनैतीक स्वतंत्रता, मानवाधिकाराची ग्वाही आणि आत्मसन्मानाची विविध तत्व होते.UNDP चा मानव विकास रिपोर्ट हा तीन तत्वांचे संमिश्रण आहे. ते म्हणजे -

1) जन्माच्या वेळी जीवन संभाव्यता.

2) शिक्षणाचा स्तर. (प्रौढ शिक्षणाचा दर, प्राथमिक,माध्यमिक,उच्च शिक्षणाचा दर)

3) जीवनाचा स्तर. (USD वर आधारीत प्रती व्यक्ति GDP)

या तीन तत्वांचे अधिकतम आणि न्युनतम मुल्याच्या आधारे HDI निर्देशांकाची सरासरी आखली जाते. या रिपोर्ट नुसार भारत सन 2006 मध्ये HDI निर्देशांकाच्या कमवारीत 126 व्या कमांकावर होता. सन 2008 मध्ये UNDP चे भारतातील प्रतीनिधी मैक्सिन ओल्सन व योजना आयोगाचे उपाध्यक्ष मोटेक सिंह अहलूवालीया यांनी दिल्ली येथे मानव विकास रिपोर्ट प्रकाशीत केला, यात भारत देश 128 (Value 0.619) व्या 1 | P a g e 
कमांकावर आहे. सन 2006 च्या तुलनेत भारत दोन कमांकाने खाली घसरलेला असल्याचे दिसून येतो.

UNDP प्रमाणे भारत सरकारद्वारे योजना आयोगाच्या सहाय्याने राष्ट्रिय मानवीय विकास रिपोर्ट प्रकाशित करण्यात येतो. सन 2001 मध्ये मानवीय विकास रिपोर्ट प्रकाशित करण्यात आला. योजना आयोगाने राष्ट्रिय मानवीय विकास रिपोर्ट तयार करतांना UNDP चे HDI सुचकांपेक्षा वेगळया नविन सुचकांचा आधार यात घेतला.

सारणी क. 1

\begin{tabular}{|l|l|}
\hline \multicolumn{1}{|c|}{ मुख्य घटक } & \multicolumn{1}{|c|}{ राष्ट्रिय मानवीय विकास रिपोर्ट चे निर्देशक } \\
\hline 1) जीवन संभाव्यता & $\begin{array}{l}\text { 1) } 1 \text { वर्षापर्यंत जीवन संभाव्यता. } \\
\text { 2) बाल मृत्यु दर. }\end{array}$ \\
\hline 2) शैक्षणीक प्रगती & $\begin{array}{l}\text { 1) } 7 \text { वर्ष आणि पेक्षा जास्त वयाचे साक्षरता } \\
\text { दर. } \\
\text { 2) औपचारिक शिक्षणाची गहनता. }\end{array}$ \\
\hline 3) आर्थिक प्रगती & 1) प्रती व्यक्ति वास्तविक उपभोग व्यय. \\
\hline
\end{tabular}

नोट- भारतीय अर्थव्यवस्था, दत्त आणि सुंदरम, 2009 पृ.48

2001 च्या रिपोर्ट नुसार अंदमान आणि निकोबार बेट प्रथम स्थानावर असुन महाराष्ट्र राज्य 15 व्या तर बिहार राज्य सर्वात खालच्या (32) स्थानावर आहे.

\section{सरकारी योजना आणि मानवीय विकास}

भारतात आतापर्यंत 10 पंचवार्षीक योजना पुर्ण झाल्या असून सध्या 'अधिक तीव्र आणि समावेशी विकासाकडे' असे ब्रिद वाक्य असलेली 11 वि पंचवार्षीक योजना सुरू आहे. प्रस्तुत शोधनिबंधात या पंचवार्षीक योजनेच्या आधारे मानवी विकासाची प्रगती तपासण्याचा प्रयत्न करण्यात आलेला आहे. 


\section{1) स्वास्थ ::-}

2001 च्या जनगणनेच्या आधारे भारताची लोकसंख्या 102.7 करोड आहे.

15 फेब्रुवारी 2000 ला राष्ट्रिय लोकसंख्या नितीची घोषणा करण्यात आली त्यानुसार भारताने सन 2046 पर्यंत लोकसंख्या स्थिर करण्याचा संकल्प घेतला आहे. सन 2026 पर्यंत ही लोकसंख्या 140 करोड होण्याची शक्यता यात वर्तविण्यात आली. UNDP चा मानव विकास रिपोर्ट हा जन्माच्या वेळी जीवन संभाव्यता या घटकाचा आधार घेतो. भारत सरकारने सन 1952 पासून लोकसंख्या नियंत्रणाला सुखूवात केली. आरोग्याचा सबंध व्यक्ति आणि समाज जीवनाशी संबंधित असल्याने सरकारने 11 व्या पंचवार्षीक योजनेत सार्वजनिक स्वास्थ्या करिता $12,0,879$ करोड रू. खर्च करण्याचे ठरविले आहे. तसेच 2010-11 च्या केंद्रीय अर्थसंकल्पात आरोग्य आणि कुटुंब कल्याणाकरीता 22,300 कोटी आणि आरोग्य विम्याकरीता 1 लाख स्मार्ट कार्डाची तरतूद करण्यात आलेली आहे.

2001 च्या राष्ट्रिय मानविय विकास रिपोर्ट नुसार भारतात जन्माच्या वेळी जीवन संभाव्यता 60.7, अर्भक मृत्यू प्रमाण दर हजारी 115 आणि 5 वर्षखालील बालमृत्यु 98 एवढी दर्शविण्यात आली आहे. भारतात सध्या बाल मृत्यूचे प्रमाण 63 प्रती हजार तर प्रजनन काळात स्त्री मृत्यूचे प्रमाण 540 प्रती हजार आहे. कुपोषीत बालकांचे प्रमाण भारतात सध्या $26 \%$ आहे. कुपोषणाची समस्या गेल्या अनेक वर्षापासून देशाला भेडसावत आहे. डॉ. अभय बंग समीतीच्या अहवालानुसार दरवर्षी 1 लाख 30 हजार बालके मृत्युमूखी पडत असुन यातील 82 हजार बालके ग्रामिण भागातील आहेत. महाराष्ट्रातील बाल मृत्यू चे प्रमाण 42 प्रती हजार एवढे आहे.

महाराष्ट्र सरकारच्या मानव विकास 2002 च्या रिपोर्ट नुसार :-

भारतात दर 1 लाख लोकसंख्येमागे $1.6 \%$ दवाखाने, $69.1 \%$ बेडची व्यवस्था व $3 \%$ औषधालयाची व्यवस्था आहे. यातील ग्रामीण भागात $0.7 \%$ दवाखाने, $19.4 \%$ बेडची 
व्यवस्था आणि $1.7 \%$ औषधालयाची व्यवस्था आहे. भारतातील खया स्वास्थ व्यवस्थेचे उत्तम उदाहरण म्हणजे अनुसूचित जारीं मध्ये दररोज 100 मुलांचा जन्म होतो त्यापैकी 12 मुल आपल्या पाचव्या वाढदिवसापर्यंत ही जगू शकत नाही. येण्याय्या काळात भारताला स्वास्थ समस्या ही सर्वात मोठी समस्या असणार आहे.

सध्याचा काळ हा जागतिकीकरणाचा काळ असून यात सरकारने 2005 मध्ये नविन पेटंट कायदा तयार केला, जो 5 मे 2006 पासून सुधारित कायदा म्हणून प्रभावीत झाला आहे. श्री. बी.के. केयाल, संयोजक - पेटंट कायदा कार्यदल यांच्या मते, नविन पेटंट कायदयात $70 \%$ औषधांचा समावेश होणार आहे. परिणामतः बौद्धिक संपदा अधिकाराच्या प्रभावातून पेटंटधारकाला मोठी रक्कम दयावी लागणार आहे. याचा एकंदरित प्रभाव किंमतवाढीवर होवुन औषधांच्या किंमती 5 ते 10 पटीने वाढण्याची शक्यता वर्तविण्यात आली. याचा सर्वाधिक जास्त परिणाम ग्रामिण गरिब जनतेवर होणार आहे.

\section{२) शिक्षण ::-}

UNDP चा मानव विकास रिपोर्ट हा शिक्षणाचा स्तर (प्रौढ शिक्षणाचा दर, प्राथमिक, माध्यमिक, उच्च शिक्षणाचा दर) या दुसय्या घटकावर आधारलेला आहे. भारतीय संविधानाच्या कलम 14 नुसार 14 वर्षापर्यंतच्या मुलामुलींना शिक्षण मोफत आणि सक्तिचे देण्याची जबाबदारी ही शासनाची आहे. भारत सरकारने 11 व्या पंचवार्षीक योजनेत शिक्षणाकरिता एकूण $11,02,327$ करोड रू. ची तरतूद केली आहे, जी एकूण खर्चाच्या $9.5 \%$ आहे. यात 3,675 करोड रू. प्राथमिक शिक्षणाकरिता आणि 3,136 करोड रू. शालेय शिक्षणाकरिता खर्च करण्याची तरतुद करण्यात आलेली आहे. प्राथमिक शिक्षणासाठी शासन सध्या प्रती विद्यार्थी 984 रू. खर्च करीत आहे. जगात शिक्षणावर खर्च करण्याच्या कमवारीत देशाचा 115 वा क्रमांक लागतो. 
11 व्या पंचवार्षीक योजनेत शिक्षणाकरिता काही मुख्य उददेश निर्धारीत करण्यात आले आहे. :-

1) शाळा सोडण्याचे प्रमाण $52.2 \%$ वरून $20 \%$ पर्यंत आणने.

2) 0-7 या वयोगटातील साक्षरता दर सन 2012 पर्यंत $85 \%$ करने.

3) साक्षरतेतील लैगिक अंतर $10 \%$ पर्यंत कमी करने.

4) उच्च शिक्षणाला बाहेर जाणान्यांचे प्रमाण $10 \%$ वरून $15 \%$ पर्यंत वाढविने .

2001 च्या राष्ट्रिय मानविय विकास रिपोर्ट नुसार भारताचा साक्षरता दर हा $65.20 \%$ आहे. यात $75.64 \%$ पुरूष साक्षरता तर $54.3 \%$ स्त्री साक्षरता दर्शविण्यात आलेली आहे. यात अनुसूचित जातील लोकांच्या साक्षरतेचा दर $37.4 \%$ आणि अनु.

जमाती मधील लोकांच्या साक्षरतेचा दर $29.6 \%$ आहे. 7 ते 14 या वयोगटातील $71.44 \%$ मुले आणि $56.23 \%$ मुलीं साक्षर आहेत याचा अर्थ असा की, अजूनही $39.42 \%$ मुले आणि $43.77 \%$ मुलींपर्यंत अजुनही शिक्षण पोहचलेले नाही. वर्ग 1 ते 5 पर्यंत शाळा सोडण्याचे प्रमाण मुलांमध्ये $38.23 \%$, मुलींमध्ये $41.34 \%$ एवढे आहे. वर्ग 1 ते 10 पर्यंत शाळा सोडण्याचे प्रमाण मुलांमध्ये $65.44 \%$, मुलींमध्ये $70.22 \%$ एवढे आहे. भारताचा विचार करता 15 वर्षाखालची $25 \% \mathrm{OBC}$ संवर्गातील मुले शाळेतच जात नाहीत. भारतात प्रती 1 हजार लोकसंख्येवर शाळांची संख्या पाहता प्राथमिक शाळा $5.4 \%$ आणि उच्च प्राथमिक शाळा $2.75 \%$ आहेत. 15 वर्षावरील प्रौढ साक्षरता पाहता पुरूषात $61.89 \%$ आणि स्त्रीया मध्ये $34.9 \%$ एवढी आहे. ग्रामीण भागात प्रौढ साक्षरता पुरूषात $54.89 \%$ आणि स्त्रीया मध्ये $24.92 \%$ आहे जे एकूण प्रौढ साक्षरतेच्या $40.34 \%$ आहे. 2001 च्या राष्ट्रिय मानविय विकास रिपोर्ट नुसार महाराष्ट्राचा 15 वा क्रमांक आहे. महाराष्ट्रात करण्यात आलेल्या सर्वेक्षणानुसार प्राथमिक शिक्षणाची धक्कादायक माहिती समोर आलेली आहे. 
1) राज्यातील सरकारी शाळेतील 8 लाख विद्यार्थी अजुनही अशिक्षित आहेत.

2) चवथ्या वर्गातील $38 \%$ विद्याथ्यांना दुसय्या वर्गाचा परिच्छेद वाचता येत नाही.

3) आजचा दिवस कोणता? याचे उत्तर न देता येणारे $6,53,410$ विद्यार्थी होते.

4) 1 ते 10 पर्यंतचे इंग्रजीत पाढे न येणारे $6,04,540$ विद्यार्थी होते .

5) तिसरीच्या विद्यार्थांना 6 गुणीले 4 विचारले असता 5,20,240 विद्यार्थ्यांना उत्तर देता आले नाही.

6) पाचवी च्या विद्यार्थांना भारत केक्हा स्वतंत्र झाला असे विचारले असता $2,87,863$ विद्यार्थीना उत्तर देता आले नाही.

7) सहावी च्या विद्यार्थीना एडीसनने लावलेल्या शोधाचे नाव विचारले असला $2,58,874$ विद्यार्थीना उत्तर देता आले नाही.

याचाच अर्थ एकीकडे शहरात इंटरनेट सर्फींग करणायांची संख्या वाढत असली तरी दुसरीकडे इंग्रजीत पाढेही न येणारे विद्यार्थी ही आहेत याची सरकारने विशेष दखल घ्यावी.

3) जीवन स्तर ::-

UNDP चा मानव विकास रिपोर्ट जीवनाचा स्तर (USD वर आधारीत प्रती व्यक्ति GDP) या तिसया घटकावर आधारलेला आहे. जीवनाचा स्तर हा व्यक्तिला प्राप्त होत असलेल्या सोयी आणि सुविधांवर आधारीत आहे.

11 व्या पंचवार्षीक योजनेत भारतातील जीवनमानाचा स्तर उंचावण्याकरीता विविध तरतूदी केलेल्या दिसुन येतात. :-
(1) पाणी पुरवठा $1,28,272$ क.रू
(2) गृह निर्माण 26,007 क.रू

(3) अन्य सामाजिक सेवा $2,71,872$ क.रू.

2010-11 च्या केंद्रीय अर्थसंकल्पात या करीता :-

(1) झोपडवासी विकासाकरीता 1,270 क.रू. (2) इंदिरा आवास योजना 10,000 क.रू. 
(3) पिण्याचे पाणी 10,580 क.रू.

2001 च्या राष्ट्रिय मानविय विकास रिपोर्ट नुसार भारतातील जीवनाचा स्तर :-

1) भारतातील दारिद्रय रेषेचे प्रमाण $26.10 \%$ एवढे आहे. यातील ग्रामीण भागातील प्रमाण $27.09 \%$ आणि $23.62 \%$ शहरी भागातील आहे.

2) भारतात पक्क्या संडासाचे प्रमाण $49.32 \%$ आहे. यातील अनु. जातीमध्ये $11.06 \%$ तर अनु. जमातीमध्ये $7.22 \%$ आहे.

3) शुद्ध पिण्याच्या पाण्याचे प्रमाण $62.30 \%$ आहे. यात ग्रामीण भागातील प्रमाण $55.54 \%$ आणि $81.38 \%$ शहरी भागातील आहे. यातील अनु. जातीमध्ये $63.60 \%$ तर अनु. जमातीमध्ये $43.21 \%$ आहे.

4) विद्युत कनेक्शनचे भारतातील प्रमाण $42.37 \%$ एवढे आहे. यातील अनु. जातीमध्ये $28.10 \%$ तर अनु. जमातीमध्ये $22.80 \%$ आहे.

पक्के संडास, शुद्ध पिण्याचे पाणी आणि विद्युत कनेक्शन सुविधा नसलेले भारतातील प्रमाण अनु. जातीमध्ये $28.06 \%$; अनु. जमातीमध्ये $45.30 \%$ आणि अन्य $21.37 \%$ इतके आहे.

5) ग्रामीण पातळीवर रस्ते विकासाचे प्रमाण 1,500 लोकसंख्या असलेल्या भागात $78.04 \%$ तर 1,000 पेक्षा कमी लोकसंख्या असलेल्या भागात $49.18 \%$ आहे. वरील आकडेवारी ही भारताची वास्तविकता दर्शविणारी आहे यावसून आणखी सोयी सुविधेची गरज भारतीय समाज जीवनाला आहे याची जाणिव होते, त्यातच ग्रामीण भागावर प्राथमीक स्वसूपात भर देण्याची गरज आहे.

ग्रामीण भागात पुरुषांच्या बेरोजगारीचे प्रमाण 1993 - 94 मध्ये $5.6 \%$ वरून 2004 मध्ये $9.0 \%$ तर महिला बेरोजगारीचा विचार केल्यास ग्रामीण भागात 1993-94 मध्ये $5.6 \%$ वरून 2004 पर्यंत $9.3 \%$ पर्यंत वाढले आहे. 
ग्रामीण रोजगारीत एका अंदाजानुसार एकूण कृषी मजुरांच्या $80 \%$ मजुर हे अनुसूचित जातीचे आहेत. यातही मिळणारी मजुरी ही महाराष्ट्राचा विचार करता 2006-07 दरम्यान 45 रू. होती.

भारतात बेरोजगारीचा स्तर वाढत चालला आहे, यातच राष्ट्रिय रोजगार गारंटी योजने करिता अर्थसंकल्पात 40,100 क.रू. ची तरतूद करण्यात आलेली आहे. सन 2002 पर्यंत भारतात $18.5 \%$ करोड लोक बेरोजगार होते यातील 1993-2004 या दहा वर्षात जागतिकीकरणाच्या काळात $40 \%$ बेरोजगारांची भर पडली आणि याच काळात सरकारने $12.45 \%$ नोकरीतील पदे समाप्त करून टाकले. बाल श्रमाच्या बाबतीत सांगावयाचे झाल्यास जगाच्या पाठीवरील दर चवथा बालक हा भारतीय बाल कामगार आहे तर भारतातील दर तिसया कुूूंबातील बालक हा बाल कामगार म्हणून काम करतो आहे. आज घडीला भारतात 10 करोड बाल श्रमीक आहेत.

\section{समारोप :-}

सन 2020 पर्यंत भारत महासत्ता होण्याची स्वप्न पाहतो आहे. सन 2006 मध्ये भारत HDI च्या बाबतीत 126 व्या क्रमांकावर होता. सन 2008 मध्ये घसरून 128 व्या क्रमांकावर आला. भारताची ही घसरन भारतात जीवन संभाव्यता ,आर्थिक प्रगती, शैक्षणीक प्रगती च्या बाबतीत अजुनही अन्य विकसित देशाच्या तुलनेत मागेच आहे असे दर्शविते. तेक्हा भारताला HDI च्या क्रमवारीत वरच्या स्थानावर येण्याचे असल्यास विकासाच्या एकंदरीत सर्व पैलूंवर भर द्यावा लागणार आहे.

भारताचा GDP वाढतो म्हणून भारत विकसीत होत आहे असे नाही तर जेक्हापर्यंत झालेला विकास हा समाजाच्या अंतीम घटकापर्यंत पोहचत नाही आणि त्याचे सर्व नागरीकांचे मानवाधिकाराचे सर्व हक्क अबाधित राहत नाही तेक्हापर्यंत विकासाची संकल्पना ही इंडीया पुरतीच मर्यादित राहणार आहे. 


\section{संदर्भ :-}

1) UNDP (2007-08-) : Human Development Report Pub. By United Nations Development Programme New York ,10017, USA.

2) Planing Commission (2001) : National Human Development Report Gov. Of India

3) Gov. Of Maharashtra (2002) : Human Development Report Maharashtra Mumbai .

4) Thorat Sukhadeo (2009): Dalit In India , SAGA Publications India Pvt. Ltd. Mathura road, New Delhi,

5) दत्त स्वद्र, सुंदरम के.पी.एस. (2006) : भारतीय अर्थव्यवस्था, एस.चंद आणि कंपनी प्रकाशन, रामनगर, दिल्ली.

6) संपादक - योजना (2010) : (अर्थसंकल्प विशेष अंक 2010-11) Vol. XXXVII No. VIII ,मार्च

7) खंडेला मानचंद (2006) उदारीकरन और भारतीय अर्थव्यवस्था, आविष्कार पब्ली. जयपूर.

8) सोनकांबळे अशोक (2009) : आरोग्य प्रशासन आणि कुपोषीत बालके, IPRA Vol. III जाने - मार्च

9) चक्हाण शांताराम (2009) : शिक्षण प्रणाली : धोरण, समस्या व उपाय IPRA Vol. III जाने - मार्च

10) काबरा कमल नयन (2009) : वैकल्पिक आर्थिक वार्षिकी, भारत 2008-09, दानिश बुक प्रकाशन, गाजीपूर गॉव, दिल्ली,

11) संपादक- साहित्य भवन प्रतियोगिता सीरीज (2006) भारतीय अर्थव्यवस्था एक दृष्टि में,साहित्य भवन पब्लिश एण्ड डिस्ट्रीब्यूटर्स, आगरा. 